\title{
Unsplittable Anycast Flow Problem: Formulation and Algorithms
}

\author{
Krzysztof Walkowiak \\ Chair of Systems and Computer Networks, Faculty of Electronics, \\ Wroclaw University of Technology, Wybrzeze Wyspianskiego 27, 50-370 Wroclaw, Poland \\ Tel.: (+48)713203539; Fax.: (+48)713202902 \\ Krzysztof.Walkowiak@pwr.wroc.pl
}

\begin{abstract}
Our discussion in this article centers on a new optimization problem called unsplittable anycast flow problem (UAFP). We are given a directed network with arc capacities and a set of anycast requests. Anycast is a one-to-oneof-many delivery technique that allows a client to choose a content server of a set of replicated servers. In the context of unsplittable flows, anycast request consists of two connections: upstream (from the client to the server) and the downstream (in the opposite direction). The objective of UAFP is to find a subset of the requests of maximum total demand for which upstream and downstream connection uses only one path and the capacity constraint is satisfied. To our best survey, this is the first study that addresses the UFP (unsplittable flow problem) in the context of anycast flows. After formulation of UAFP, we propose several heuristics to solve that problem. Next, we present results of simulation evaluation of these algorithms.
\end{abstract}

Keywords: UFP, connection-oriented network, anycast.

\section{Introduction}

In this paper we consider a new version of the Unsplittable Flow Problem (UFP) for anycast flows. Anycast is a one-to-one-of-many technique to deliver a packet to one of many hosts. Anycast paradigm becomes popular, since there is a need to facilitate the distribution of popular content (electronic music, movies, books, software) in the Internet. One of exemplary techniques that apply anycast traffic is Content Delivery Network (CDN). For more details on anycast flows and CDNs refer to [3], [8-10]. In this work we consider on anycast unsplittable flows. Several connection-oriented (co) techniques apply unsplittable flows, e.g. MultiProtocol Label Switching (MPLS), Asynchronous Transfer Mode (ATM), optical network [2]. In c-o networks an anycast demand consists of two connections: one from the client to the server (upstream) and the second one in the opposite direction (downstream). Upstream connection is used to send user's requests. Downstream connection carries requested data. Consequently, each anycast demand is defined by a following triple: client node, upstream bandwidth requirement and downstream bandwidth requirement. In contrast, a unicast demand is defined by the following triple: origin node, destination node and bandwidth requirement. According to observations of real networks and user behavior, 
there is asymmetry in anycast flows, since usually more data is received by clients then is sent to content servers Therefore, bandwidth of upstream connection is typically much lower than bandwidth of downstream connection.

The considered unsplittable anycast flow problem (UAFP) can be formulated as follows

Given network topology, anycast traffic demand pattern, location of replica servers, link capacity

Minimize volume of un-established anycast requests

Over selection of replica server, routing (path assignment)

Subject to anycast connection-oriented flow constraints, capacity constraints

To the best of the author's knowledge, this is the first work that addresses the UFP in the context of anycast flows. The objectives of this paper are twofold: mathematical formulation of the UAFP and development of effective heuristics for the UAFP. Evaluation of proposed algorithms through simulation experiments is also provided.

\section{Related Work}

The most intuitive approach to solve unicast UFP is the greedy algorithm (GA), which proceed all connections in one pass and either allocate the processed request to the shortest path or reject the request if such a feasible path does not exist, i.e. origin and destination node of the connection do not belong to the same component of considered graph [5]. A modification of GA called bounded greedy algorithm (BGA) works as follows [5], [7]: Let $L$ be a suitable chosen parameter. Reject the request if there is no feasible path of the length at most $L$ hops. Otherwise accept the request. Another version of GA is careful BGA (cBGA) proposed in [7]. Online algorithms can also solve the UFP. Several such algorithms were developed in the context of dynamic routing in MPLS networks [6]. MPLS supports the explicit mode, which enables the source node of the LSP to calculate the path. The main goal of dynamic routing is to minimize the number of rejected calls or the volume of rejected calls. The most common approach to dynamic routing is the shortest path first (SPF) algorithm based on an administrative weight (metric).

\section{Problem Formulation}

In this section we will formulate the UAFP. We consider an offline UAFP, in which we know a priori all requests that are to be located in the network. To mathematically represent the problem we introduce the following notations

Sets:

$V$ set of vertices representing the network nodes.

$A$ set of arcs representing network directed links.

$P$ set of connections in the network. A connection can be of two types: downstream or upstream.

$\Pi_{\mathrm{p}}$ the index set of candidate routes (paths) $\pi_{p}^{k}$ for connection $p$. Route $\pi_{p}^{0}$ is a 
"null" route, i.e. it indicates that connection $p$ is not established. For upstream connection, paths are between client node and server. Analogously, downstream candidate paths connect server and client node.

$X_{r}$ set of variables $x_{i}^{k}$, which are equal to one. $X_{r}$ determines the unique set of Constants: currently selected routes.

$\delta_{p a}^{k}$ equal to1, if arc $a$ belongs to route $k$ realizing connection $p ; 0$ otherwise

$Q_{p}$ Volume (estimated bandwidth requirement) of connection $p$

$c_{a}$ capacity of arc $a$

$\tau(p)$ index of the connection associated with connection $p$. If $p$ is a downstream connection $\tau(p)$ must be an upstream connection and vice versa.

$o(\pi) \quad$ origin node of route $\pi$. If $\pi$ is a "null", then route, $o(\pi)=0$.

$d(\pi) \quad$ destination node of route $\pi$. If $\pi$ is a "null", then route, $d(\pi)=0$.

Variables:

$x_{p}^{k} \quad 1$ if route $k \in \Pi_{p}$ is selected for connection $p$ and 0 otherwise.

$f_{a}$ flow of $\operatorname{arc} a$.

The UAFP can be formulated as follows

$$
L F=\min _{X_{r}} \sum_{p \in P} x_{p}^{0} Q_{p}
$$

subject to

$$
\begin{gathered}
\sum_{k \in \Pi_{p}} x_{p}^{k}=1 \quad \forall p \in P \\
x_{p}^{k} \in\{0,1\} \quad \forall p \in P, \forall k \in \Pi_{p} \\
f_{a}=\sum_{p \in P} \sum_{k \in \Pi_{p}} \delta_{p a}^{k} x_{p}^{k} Q_{p} \quad \forall a \in A \\
f_{a} \leq c_{a} \quad \forall a \in A \\
\sum_{k \in \Pi_{p} x_{p}^{k} d\left(\pi_{p}^{k}\right)=\sum_{k \in \Pi_{\tau(p)}} x_{\tau(p)}^{k} o\left(\pi_{\tau(p)}^{k}\right)} \forall p \in P \\
\left.X_{r}=\left(\begin{array}{l}
\bigcup \\
i, k: x_{i}^{k}=1
\end{array} x_{i}^{k}\right\}\right)
\end{gathered}
$$

The objective function (1) is a lost flow $(L F)$. Function $L F$ is as a sum of all demands (connections) that are not established (variable $x_{p}^{0}$ is 1). It should be noted that also an equivalent objective function could be applied, in which we maximize the total volume of established connections. Condition (2) states that the each connection 
can use only one route or is not established. Therefore, we index the subscript of variable $x_{p}^{k}$ starting from 0 , i.e. variable $x_{p}^{0}$ indicates whether or not connection $p$ is established. If it is established, $x_{p}^{0}=0$ and one of variables $x_{p}^{k}(k>0)$, which is equal to 1 , indicates the selected path. Constraint (3) ensures that decision variables are binary ones. (4) is a definition of an arc flow. Inequality (5) denotes the capacity constraint. Equation (6) guarantees that two routes associated with the same anycast demand connect the same pair of nodes. Furthermore, (6) assures that if upstream connection is not established ( $x_{p}^{0}=0$ and the "null" route is selected) the downstream connection of the same anycast demand is not established, and vice versa. Finally, (7) is a definition of a set $X$ called a selection that includes all variables $x$, which are equal to 1 . Each selection denotes for each connection either the selected route or indicates that the particular connection is not established. Note, that we call a connection $p$ established in selection $X$ if $x_{p}^{0} \notin X$.

\section{Algorithms}

The first, quite intuitive, approach to solve UAFP can be a modification of heuristics developed for unicast UFP. Now, we shortly discuss how to adapt GA (greed algorithm) to anycast flows. We refer to this new algorithm as AGA (anycast GA). AGA proceeds all anycast requests in a one pass. Requests can be sorted accordingly to selected criterion (e.g. bandwidth requirement). For each request we perform the following procedure. First, we construct a residual network for downstream connection of considered anycast demand, i.e. we remove from the network all arcs that have less residual capacity than volume of downstream connection. Next, we find shortest paths between each server and client node. If none path exists, the demand is rejected. Otherwise, we select a shortest path among all found paths. We repeat the same procedure for upstream connection. The residual network is computed again. However, in this case we try to find a shortest path from the client node to the server node already selected for downstream connection. This follows from the asymmetry of anycast flows discussed in Section 1, because the downstream connection is more important than upstream connection.

Now we propose two new offline algorithms for the UAFP: Anycast Greedy Algorithm with Preemption (AGAP) and Anycast Greedy Algorithm with Preemption and Flow Deviation (AGAPFD). Both algorithms use the preemption mechanism, which consists in removing from the network already established connections in order to enable establishment of other connections to minimize the objective function.

We apply some temporary variables in both algorithms. Sets $H$ and $F$ are selections including decision variables $x$ equal to 1 . Sets $B$ and $D$ include indexes of connections. Operator $\operatorname{first}(B)$ returns the index of the first connection in set $B$. Operator $\operatorname{sort}(H)$ returns indexes of connections included in $H$ ordered according to their paths' length given by the metric CSPF [1] starting with the longest. We select CSPF metric due to its effectiveness in many dynamic routing problems. Operator $A G A(H, i, j)$ returns either the pair of indexes of calculated routes: downstream and upstream 
according to AGA or a pair of zeros, if a pair feasible routes does not exist for connections $i$ and $j$ associated with the same anycast demand. Operator $u e(H)$ returns indexes of connections, which are not established in $H$, while es $(H)$ returns indexes of connections established in $H$.

\section{Algorithm AGAP}

$\underline{\text { Step } 1 .}$ Let $H$ denote an initial solution, in which none connection is established. Let $B:=\operatorname{sort}(H)$.

Step 2. Set $i:=f i r s t(B)$ and $\{d, u\}:=A G A(H, i, \tau(i))$. Calculate $B:=B-\{i\}, B:=B-\{\tau(i)\})$.
a) If $d>0$ set $H:=\left(H-\left\{x_{i}^{0}\right\}\right) \cup\left\{x_{i}^{u}\right\}, H:=\left(H-\left\{x_{\tau(i)}^{0}\right\}\right) \cup\left\{x_{\tau(i)}^{d}\right\}$. Go to step 2c.
b) If $d=0$ go to step 3 .
c) If $B=\varnothing$ then stop the algorithm. Otherwise go to step 2 .

Step 3. Set $D:=\operatorname{es}(\operatorname{sort}(H))$.
a) Set $j:=f i r s t(D)$. Set $D:=D-\{j\}$ and $F:=\left(H-\left\{x_{j}^{m}\right\}\right) \cup\left\{x_{j}^{0}\right\}$, where $x_{j}^{m} \in H$. Next set $D:=D-\{\tau(j)\}$ and $F:=\left(F-\left\{x_{\tau(j)}^{m}\right\}\right) \cup\left\{x_{\tau(j)}^{0}\right\}$, where $x_{\tau(j)}^{m} \in H$.
b) Find
$\{d, u\}:=A G A(F, i, \tau(i))$
If $\quad d>0$
set
$H:=\left(H-\left\{x_{i}^{0}\right\}\right) \cup\left\{x_{i}^{u}\right\}$
$H:=\left(H-\left\{x_{\tau(i)}^{0}\right\}\right) \cup\left\{x_{\tau(i)}^{d}\right\}$ and go to step 2 .
c) If $d=0$ then go to step $3 \mathrm{~d}$.
d) If $D=\varnothing$ then go to step 2c. Otherwise go to step 3a.

The idea of AGAP is as follows. We start with an "empty" solution - none connection is established. We process connections one-by-one sorted according to selected criterion. If the AGA can find a pair of feasible paths for current anycast demand, we establish upstream and downstream connections (step 2a). Otherwise (step 2b), we go back to already established connections and preempt each of these demands (step 3a) trying to establish again considered anycast connections (step 3b-d).

\section{Algorithm AGAPFD $(\alpha, \beta)$}

Step 1. Let $X_{1}$ denote a feasible initial solution given by AGA. Sort all connections in $X_{1}$ according to their bandwidth requirements starting with the heaviest. Set $j:=1$.

Step 2. Set $H:=X_{j}$. Let $B:=\operatorname{sort}(H)$. Let $l$ denote the number of connections established in $H$. Set $k:=0$.

a) Set $i:=f i r s t(B)$. Calculate $B:=(B-\{i\}), \quad B:=B-\{\tau(i)\}), \quad F:=\left(H-\left\{x_{i}^{m}\right\}\right) \cup\left\{x_{i}^{0}\right\}$ where $x_{i}^{m} \in H, F:=\left(F-\left\{x_{\tau(i)}^{m}\right\}\right) \cup\left\{x_{\tau(i)}^{0}\right\}$, where $x_{\tau(i)}^{m} \in H$.

b) Set $H:=F$ and $k:=k+1$.

c) If $k>\alpha \cdot l$ then go to step 3. Otherwise go to step $2 \mathrm{a}$.

Step 3. Let $F:=A F D N B(H)$ be a selection of route variables calculated according to the Anycast Flow Deviation for Non-bifurcated flows algorithm [10].

Step 4. Set $H:=F$ and $B:=u e(H)$.

a) Set $i:=f i r s t(B)$ and find $\{d, u\}:=A G A(H, i, \tau(i))$. Calculate $B:=B-\{i\}, B:=B-\{\tau(i)\})$, $H:=\left(H-\left\{x_{i}^{0}\right\}\right) \cup\left\{x_{i}^{u}\right\}$ and $H:=\left(H-\left\{x_{\tau(i)}^{0}\right\}\right) \cup\left\{x_{\tau(i)}^{d}\right\}$.

b) Set $H:=F$. 
c) If $B=\varnothing$ then go to step 5. Otherwise go to step 4a.

Step 5. If $j \geq \beta$ stop the algorithm. Otherwise set $j:=j+1, X_{j}:=H$ and go to step 2 .

The algorithm has two input parameters that can be calibrated. Parameter $\alpha \in[0,1]$ is used to find set for preemption of $\alpha$ established connections. The second parameter of the GAPFD algorithm - $\beta$ - is a number of iterations for which the main loop of the algorithm is repeated. The main idea of the AGAPFD algorithm is as follows. We start with a feasible solution $X_{1}$, found by AGA. Next, in step 2 we remove from the network a number of connections. We find $\alpha \cdot l$ connections with the longest routes computed according to the CSPF metric [1] assigned to each arc, where $l$ denotes the number of established connections in a given selection. Next, we remove these connections. The major goal of preemption is to remove connections having routes using the most congested arcs. In Step 3 we re-optimize routes of established connections in order to change the allocation of arcs' flows and enable creation of as many as possible of un-established connections, what should yield improvement of UAFP objective function. We use the Anycast Flow Deviation for Non-bifurcated flows (AFDNB) algorithm proposed in [10]. Note that AFDNB uses as objective the network delay function, which includes the capacity constraint (5) as a penalty function. This guarantees feasibility of obtained selection. Furthermore, former studies shows that the delay function provides proportional allocation of network flows - more open capacity is left for other demands [4]. Since AGA processes connections sequentially, there is no chance to change the route of already established connections. Running of AFDNB in step 4 eliminates this constraint and enables re-optimization of already established routes. In Step 4 we process all un-established connections using AGA. The main loop of the algorithm (Steps 2-5) is repeated $\beta$ times.

\section{Results}

Algorithms AGA, AGAP, AGAPFD were coded in C++. The network on which we conduct our experiment consists of 36 nodes and 144 directed links [10]. We test 10 demand patterns for 5 various locations of 2, 3, or 4 content servers. Each of 10 demand patterns includes 360 anycast requests generated randomly. Due to asymmetry of anycast flows, we assume that upstream bandwidth is 0.1 of the downstream bandwidth. To compare results we apply competitive ration performance indicator. The competitive ration is defined as the difference between result obtained for a particular algorithm and the minimum value of objective function yielded by the best algorithm. For instance, if in a test consisting of simulations of various algorithms the minimum value of lost flow is 2000 and the considered algorithm yields 2500; the competitive ration is calculated as follows: $(2500-2000) / 2500=20 \%$. For presentation of aggregate results we apply the aggregate competitive ration, which is a sum of competitive rations over all considered experiments.

The first objective of numerical experiments was evaluation of various metrics and orderings for AGA. We have tested two link metrics: HOP and CSPF [1]. Demands were sorted according to the bandwidth requirement of downstream connection in decreasing and increasing order. In Table 1 we report aggregate competitive ration of AGA for these cases. We can watch that the best result provides CSPF metric and demand sorted in decreasing order. 
Table 1. Aggregate competitive ration of AGA using ordering CDLI and various metrics for experiment $\mathrm{C}$

\begin{tabular}{|c|c|c|c|c|}
\hline \multirow{2}{*}{ Servers } & Decreasing & Decreasing & Increasing & Increasing \\
\cline { 2 - 5 } & Hop & CSPF & HOP & CSPF \\
\hline \hline $\mathbf{2}$ & $3.5 \%$ & $3.0 \%$ & $152.7 \%$ & $165.3 \%$ \\
\hline $\mathbf{3}$ & $14.6 \%$ & $3.0 \%$ & $415.9 \%$ & $426.4 \%$ \\
\hline $\mathbf{4}$ & $52.8 \%$ & $7.9 \%$ & $1495.4 \%$ & $1536.6 \%$ \\
\hline
\end{tabular}

The next goal of experiments was tuning of AGAPFD. Due to initial trial runs we decided to set the number of iterations (parameter $\beta$ ) to 10 . We run simulations for the following values of parameter $\alpha=\{0.0 ; 0.05 ; 0.1 ; 0.2 ; 0.3 ; 0.4 ; 0.5 ; 0.6 ; 0.7 ; 0.8 ; 0.9$; $1.0\}$. In Fig. 1 we report comparison of results obtained for tested values of $\alpha$. Due to results presented in Fig. 1 we decide to set $\alpha=0.3$ for further simulations.

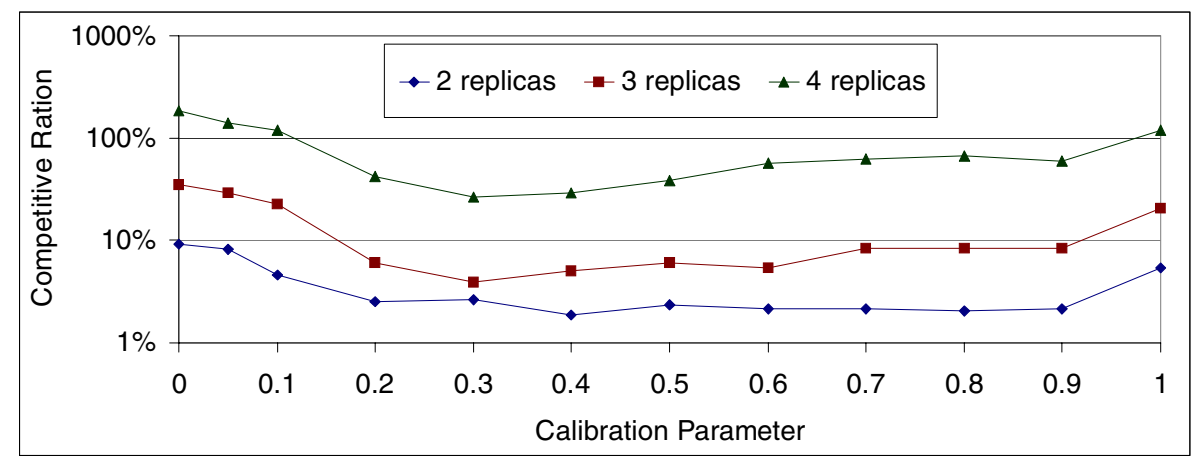

Fig. 1. Aggregate competitive ration of parameter $\alpha$ for various numbers of content servers

Finally, we run experiments to compare performance of AGA, AGAP and AGAPFD $(0.3,10)$. In Table 2 we present aggregate competitive ration for these algorithms. We can see that AGAPFD outperforms other methods - in all cases it can find the best result. The difference between AGAP and AGA increases with the number of content servers.

The main drawback of AGAPFD is the calculation time - AGAPFD needs about 25 times more decision time than AGA. Most of the time is spent on running AFDNB algorithm. AGAP needs about 3-4 times more than AGA. However, it should be noted that we consider the offline UAFP. Consequently, the decision time is not the most important criterion in our considerations.

Table 2. Aggregate competitive ration of tested algorithms

\begin{tabular}{|c|c|c|c|}
\hline Servers & AGA & AGAP & AGAPFD(0.3,10) \\
\hline \hline $\mathbf{2}$ & $6.50 \%$ & $6.02 \%$ & $0.00 \%$ \\
\hline $\mathbf{3}$ & $31.00 \%$ & $27.42 \%$ & $0.00 \%$ \\
\hline $\mathbf{4}$ & $156.64 \%$ & $112.90 \%$ & $0.00 \%$ \\
\hline
\end{tabular}




\section{Conclusion}

In this paper, we have formulated the Unsplittable Anycast Flow Problem. To our best knowledge, this is the first survey that addresses the UFP in the context of anycast flows. We have developed three heuristics solving offline version of UAFP. Through simulations we have tuned and compared the algorithms. The UAFP problem is motivated by service provider needs for fast deployment of bandwidth guaranteed services enabling fast and effective distribution of popular content over Internet. Results of our work can be applied for optimization of flows of Content Delivery Networks located in connection-oriented environment, e.g. MPLS network.

Acknowledgements. This work was supported by a research project of the Polish State Committee for Scientific Research carried out in years 2005-2007.

\section{References}

1. Crawley, E., Nair, R., Jajagopalan, B., Sandick, H.: A Framework for QoS-based Routing in the Internet. RFC2386 (1998)

2. Grover, W.: Mesh-based Survivable Networks: Options and Strategies for Optical, MPLS, SONET and ATM Networking. Prentice Hall PTR, Upper Saddle River, New Jersey (2004)

3. Hao, F., Zegura, E., Ammar, M.: QoS routing for anycast communications: motivation and an architecture for DiffServ networks. IEEE Communication Magazine, 6 (2002), 48-56

4. Kasprzak, A.: Designing of Wide Area Networks. Wroclaw Univ. of Tech. Press, (2001)

5. Kleinberg, J.: Approximation algorithms for disjoint paths problems. PhD thesis, MIT, Cambridge, (1996)

6. Kodialam, M., Lakshman, T.: Minimum Interference Routing with Applications to MPLS Traffic Engineering. In Proceedings of INFOCOM (2000), 884-893

7. Kolman, P., Scheideler, C.: Improved bounds for the unsplittable flow problem. In Proc. of the Symposium on Discrete Algorithms (2002), 184-193

8. Markowski, M., Kasprzak, A.: The web replica allocation and topology assignment problem in wide area networks: algorithms and computational results. Lectures Notes in Computer Science, Vol. 3483 (2005), 772-781

9. Peng, G.: CDN: Content Distribution Network. Technical Report, sunysb.edu/tr/rpe13.ps.gz, (2003)

10. Walkowiak, K.: Heuristic Algorithm for Anycast Flow Assignment in ConnectionOriented Networks. Lectures Notes in Computer Science, Vol. 3516 (2005), 1092-1095 